\title{
Accurate radial boundary loading and measuring methods for optical components
}

\author{
Jian Xiong ${ }^{1}$, Zhijing Zhang ${ }^{2, *}$, and Xiao Chen $^{3}$ \\ School of Mechanical Engineering, Beijing Institute of Technology, 100081 Beijing, China
}

\begin{abstract}
The two main fixing methods in the assembly process of optical components are adhesive bonding and thread fastening. For both methods, boundary loading effect is generated on optical component, which results in stress distribution in it. The quantitative prediction and control of the stress distribution in optical component is not available because the boundary load induced by adhesive or thread is difficult to control and test quantitatively. Up to now, the interrelationship and variation between the boundary load and the internal stress in optical component have not been effectively studied. Based on the above requirements, quantitative radial boundary loading and real-time measuring methods for optical component are proposed in this paper. Quantitative interrelationship between the boundary load and the stress distribution and surface form in optical component can be obtained by integrating the stress and surface measurement devices with radial boundary loading system. This innovative loading and measuring device is suitable for various sizes of optical components, and it solves the quantitative application and control problems during loading, measuring, and real-time detection of adhesive curing force. Keyword: Optical component; Stress distribution; Assembly process; Loading and measuring device; Quantitative interrelationship.
\end{abstract}

\section{Introduction}

There are two types of fixing methods in optical component assembly process: adhesive bonding and thread fastening, both of them lead to stress concentration and surface deformation in optical components [1]. Residual stress in adhesive is developed during its curing process due to the property as viscoelastic material [2]. The relationship between the quantitative influence of residual stress in adhesive and the stress field in optical components is important for the research of adhesive bonding.

Improper assembly of optical component deteriorates its surface form and degrades the imaging quality [3-6]. Adhesive bonding is one of the most common assembly methods. The bonding method, including bonding area, configuration, etc., is the main factor for the variation of residual curing force. In paper [7], the optimal configuration of the adhesive bonding was studied using finite element method (FEM).

Current research concerning stress distributions in optical components is mainly focused on stress measurements [8-11] and FEM simulations [12,13]. Since there are no

\footnotetext{
${ }^{*}$ Corresponding author: zhzhj@bit.edu.cn
} 
quantitative detection of boundary load, stress measuring data in optical component can only yield qualitative analysis for the assembly process. The magnitude of load as a boundary condition in the FEM simulations can only be set empirically, so the adjustment to the assembly process can only be qualitative or partially quantitative.

In this study, a quantitative loading method for optical components is proposed to simulate the adhesive bonding or thread fastening process. Measurements of stress distribution and surface form are carried out on optical components simultaneously during the loading process. The quantitative relationship between the boundary load and the stress distribution and surface form of optical component is revealed, which provides an effective measurement and experimental method to optimize the assembly process and improve the imaging quality of optical component.

\section{General design of the radial quantitative boundary loading and real-time measuring device for optical components}

The radial quantitative boundary loading and real-time measuring device for optical components includes: optical component (1), measuring system (2), force application system (3), and force data acquisition system (4). The force application system is located at the circumferential direction of the optical component, and the configuration of the force application system (3) is decided according to the required loading quantity and loading position.

A high-precision radial boundary loading and measuring device composed of three force application systems is taken as an example, as shown in Fig. 1, which illustrates the function and the interrelationship of each component system of the device.

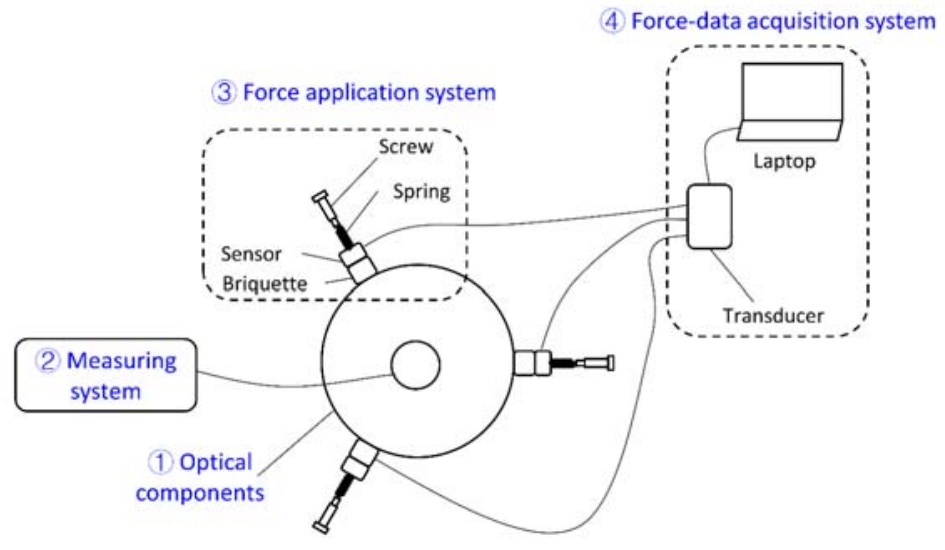

Fig. 1. Schematic diagram of the radial quantitative boundary loading and real-time measuring device for optical components.

The general design of the radial quantitative boundary loading and real-time measuring device is as follows:

In the device, high-precision radial quantitative boundary load is applied to the optical component (1) through the force application system (3). The force data acquisition system (4) detects the magnitude of the real-time load generated in the force application system (3), so load accuracy in the force application system (3) can be guaranteed. The measuring system (2) measures the stress distribution and the surface form of optical component with quantitative load. 
The function and the interrelationship of each component system of the device is described in detail in the following part.

(1) Optical component (1) is the sample to be loaded and measured.

(2) The force application system (3) generates a high-precision quantitative radial boundary load, and the radial load is applied to the optical component (1) through briquette. Besides, the force application system (3) can also measure and output the magnitude of realtime load in each force application system.

(3) Force data acquisition system (4) collects the measuring data from the high-precision sensor in force application system (3) by a multi-channel transducer. The transducer collects and amplifies the micro-voltage signal from high-precision sensors, and force data is output.

(4) Measuring system (2) measures stress distribution and surface form of the optical components (1) after load application. Measurement devices adopted in the measuring system (2) is chosen according to the actual research demands, e.g., a stress-testing instrument can be used for stress measurement, and an interferometer can be used for surface form measurement.

(5) According to the stress and surface form data obtained from measuring system (2), the magnitude of the radial load generated in the force application system (3) can be adjusted.

(6) The device can also be used for real-time measurement of dynamic changes in adhesive force during its curing process, bonding area and the number of glue points are set according to specific research requirement.

(7) Bonding or loading area are determined according to the contact area between briquettes and optical components, and the configuration of adhesive bonding or active boundary loading can be flexibly adjusted as 2 points, 3 points, 6 points or any other multiple points.

\section{The principle of quantitative radial boundary loading on optical components}

Quantitative radial loading process of optical components is achieved by multiple force application systems (3), the setting of load magnitude in force application system (3) is determined by combining the data from force data acquisition system(4) and measuring system (2). The number and installation position of the force application system can be flexibly configured according to the specific loading requirement. Fig. 2 shows the structural diagram of force application system(3).

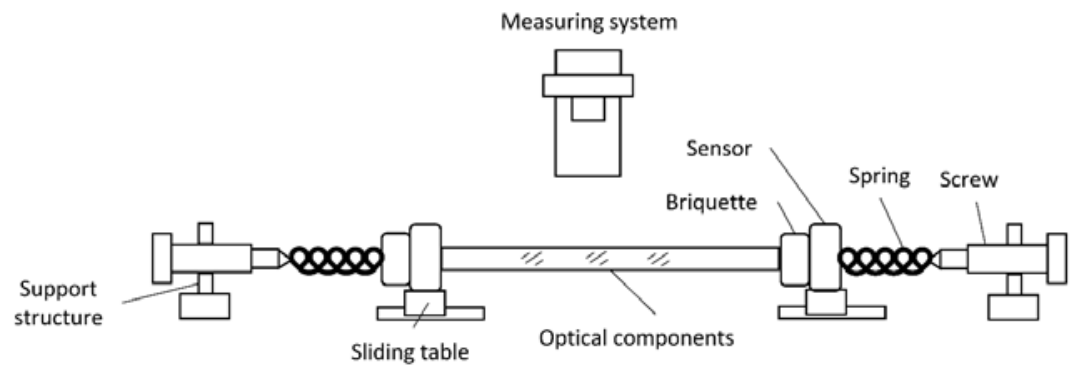

Fig. 2. The structural diagram of force application system. 
As shown in Fig. 2, force application system (3) includes: screw, spring, high-precision sensor, briquette, sliding table, and support structure. The screw is in the threaded hole on the support structure, when the screw is screwed in the centripetal direction of the optical component, it compresses the spring and generates force. The high-precision sensor is installed on the sliding table and it can slide linearly along the radial direction of the optical components; the high-precision sensor measures and transmits the force generated by the spring, this force is finally applied uniformly to the target loading area on optical component through the briquette. In addition, the sliding table that the sensor installed can reduce the friction during the loading process, so as to ensure the measuring accuracy of the sensor, the other merit of sliding table is that its linear sliding can improve the accuracy of loading direction.

Loading steps are as follows.

(1) Place the optical component in the center position, and keep screwing in the screw to make briquette contact the optical component.

(2) Continue screwing in the screw to compress the spring, sensor measures and transmits the force generated by the spring.

(3) Observe the magnitude of the real-time dynamic radial load in force data acquisition system (4), stop screwing in when load magnitude in the acquisition system stabilizes to the target value.

(4) A nut can be screwed into the screw to locked it, so as to keep load in the force application system stable at the desired value.

(5) According to the data obtained from measuring instruments, such as stress distribution or surface form, the magnitude, quantity and contact area of load in force application system (3) can be adjusted, so as to achieve the desired stress distribution or surface form in the optical component(1).

\section{Interrelationship between the boundary load and the stress distribution and surface form in optical components}

The above-mentioned quantitative radial boundary loading and real-time measuring device is adopted to investigate the interrelationship between the boundary load and the stress distribution and surface form in optical components. The relationships between loading, measurement, and FEM simulation in optical components are presented in Fig. 3.

(1) In force application system, screw compresses spring to generate the load; highprecision sensor measures and transmits the load; finally the load is applied to the optical components through briquette.

(2) In measuring system, stress distribution and surface form is measured for optical component with boundary load, so as to obtain the interrelationship between the boundary load and the stress distribution and surface form in optical component.

(3) According to FEM simulation, numerical results of stress distribution and surface form can be derived and compared with experimental results from the optical component with boundary load.

(4) In addition, adhesive bonding test can be carried out on the device, where the briquette in force application system will be replaced by adhesive. Sensors in force application system measure the dynamic force data during the curing process of adhesive. Force data acquisition system collects and stores the dynamic data of curing force, and the measuring system measures the dynamic stress distribution and surface form of optical component under dynamic curing force. By combining the real-time data of the curing force in adhesive and the stress distribution and surface form in optical component, their interrelationship can be given. 
(5) Once the curing process is completed for adhesive, force data acquisition system gives the final magnitude of curing force, and this final curing force can be used to adjust the magnitude of load in the boundary loading experiment.

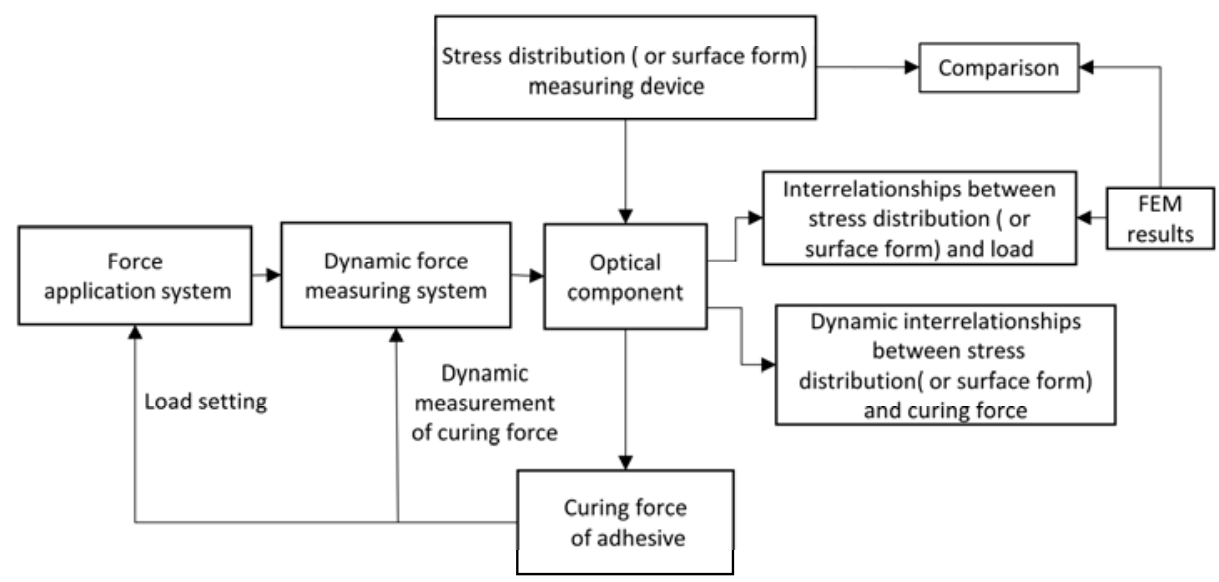

Fig. 3. Block diagram of the relationships between loading, measurement and simulation in optical components under the quantitative loading and measuring test.

\section{Experimental verification}

Experiment verification is carried out on an optical lens with diameter $120 \mathrm{~mm}$. Quantitative radial boundary loading and measuring device with six force application systems is adopted for this experiment. Load in each force application system is set as $180 \mathrm{~N}$. Test instrument in measuring system is Ilis strainMatic to obtained the stress distribution in optical lens. Besides, FEM simulation is carried out on optical lens under the above experimental condition to compare with the experimental results.

As shown in Fig. 4, Fig. 4 a) shows the stress nephogram in optical lens measured by Ilis strainMatic, Fig. 4 b) shows the stress nephogram obtained by FEM simulation. The maximum stress in Fig. 4 a) is $0.75 \mathrm{Mpa}$, the maximum stress in Fig. 4 b) is $0.797 \mathrm{Mpa}$.

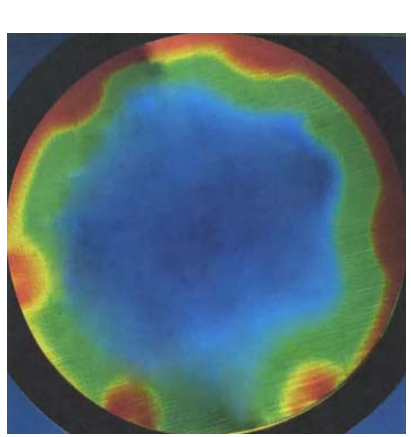

a)

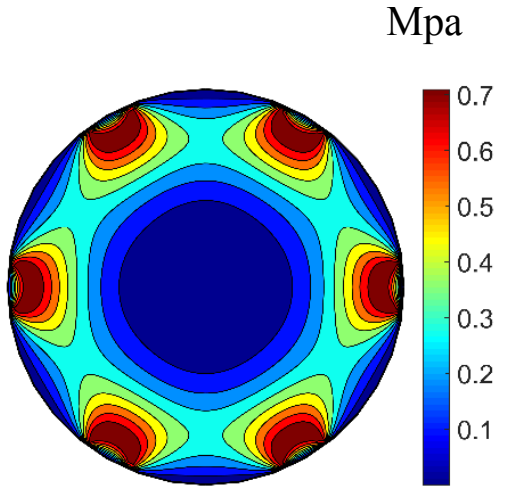

b)

Fig. 4. The stress nephogram obtained from a) Ilis strainMatic, b) FEM simulations. 


\section{Experimental results and conclusions}

The above experimental verification indicates that the difference between the experimental results and the FEM results of maximum stress in optical lens is less than $6 \%$, which verifies the validity and the accuracy of this quantitative radial boundary loading and measuring method.

This paper proposes a quantitative radial boundary loading and real-time measuring method, which can be used to study the quantitative interrelationship between the boundary load and the stress distribution and surface form of optical components. Based on the above loading and measuring method, a integrated device is developed, including the accurate radial boundary loading device and the stress or surface form measuring instrument. With this device, the stress in optical component can be quantitively and effectively controlled.

\section{Acknowledgement}

We would like to acknowledge the support of the National Natural Science Foundation of the People's Republic of China (U1937603).

\section{Reference}

1. H. Kihm, H.S. Yang, I.K. Moon, Y.W. Lee, J Opt Soc Korea 13, 201-205(2009)

2. M. Pressera and P. L. Geiss, Procedia Eng. 10, 2743-2748 (2011)

3. M. Fukuzawa, R. Kashiwagi, M. Yamada, International Conference on Indium Phosphide and Related Materials (2010)

4. M. Gstalter, G. Chabrol, A. Bahouka, L. Serreau, J.L. Heitz, G. Taupier, et al, Appl Phys A Mater Sci Process 123, 713-718(2017)

5. D. Li, C. Bi, Y. Jiang, J. Zhao, Optical Design and Testing VII (2016)

6. P.R.G. Fernandes, J.N. Maki, L.B. Gonçalves, B.F. De Oliveira, H. Mukai, Phys Rev E 97, 1-5(2018)

7. F. Maamar and A. Boudjemai, Adv. Sp. Res. 65, 2263-2275 (2020)

8. V.L. Genberg, G.J. Michels , K.B. Doyle, Proceedings of SPIE - The International Society for Optical Engineering, 24-33 (2002)

9. K. Doyle, J. Hoffman, V. Genberg, et al, International Optical Design Conference. International Society for Optics and Photonics (2002).

10. X. Shilei, L. Bincheng, C. Hao, W. Jing, Optics Letters 43, 843 (2018).

11. K. Milenko, S. Pissadakis, G. Gkantzounis,, A. Aluculesei, G. Fytas,, Acs Omega 2, 9127-9135 (2017).

12. H. Niu, Y.X. Niu, J. Li, Optical Engineering 56, 024104 (2017).

13. H. Ueno, R. Arakane, Y. Matsumoto, T. Tsumura, A. Kitazaki, T. Takahashi, S. Hirao, Y. Ohga, T. Harada, Molecules 23, 757 (2018). 